\title{
Exploiting the ACCuracy-ACCeleration tradeoff: VINS-assisted real-time object detection on moving systems
}

\author{
Betty Le Dem ${ }^{1}$, Kazuo Nakazawa ${ }^{2}$
}

\begin{abstract}
In recent years, Convolutional Neural Networks (CNNs) have repeatedly shown state-of-the-art performance for their accuracy in the task of object detection, but their heavy computational costs impede their ability for real-time detection when the supporting system is moving especially accelerating. At the same time, recent progress on visual inertial systems takes great advantage of movement information to robustly estimate the robot state and surrounding. This paper proposes to exploit the advantages of inertial odometry research for the purpose of real-time object detection system on mobile robots. We combine a CNN detector with VINS-Mono, a moving visual odometry system, and show reliable improvement in the detection process, especially as the robot accelerates or decelerates. Our system is ready-to-use in that it has very low deployment cost and requires no calibration. The resulting system allows for simultaneous robot state estimation and object detection, as well as object tracking. Lastly, the architecture is flexible in that it is not restrained to a specific object type or detector.
\end{abstract}

\section{INTRODUCTION}

For a robot to accomplish an ever-growing number of intricate tasks, understanding the surrounding environment it is moving through is of paramount importance. The task of object detection has seen a surge of progress since the introduction of deep convolutional neural networks. Given a large enough amount of training samples, most architectures can detect targeted objects. You Only Look Once [1] is one of the fastest architecture and has been applied successfully in context with very limited computational resources [4][2]. Although these systems have strong performances on fixed camera configurations, it comes at a cost of sub-real time processing rate (around 3 to $4 \mathrm{fps}$ for reliable results) which causes noise, detection inaccuracy or offsets in the case of a moving support. This is a phenomenon we decided to call the Accuracy-Acceleration tradeoff.

Another field of research has produced important development: Visual Inertial Odometry (VIO). It takes advantage of the robot moving conditions to infer its position with camera and inertial sensors. These inertial sensors retrieve the acceleration and angular information for a state prediction, and their estimation are corrected with visual features; the more the system moves the more accurate is the inference, inversely compared with $\mathrm{CNN}$ detection. In the case of monocular camera, Visual-Inertial State Estimator (VINSMono) [20] is one of the state-of-art system as stated in

\footnotetext{
1 Pr. Nakazawa Laboratory, Department of System Design Engineering, Faculty of Science and Technology, University of Keio, 223-8522 Yokohama, Japan betty.le.dem@keio.jp, betty.le.dem@gmail.com ,https://github.com/bledem/

${ }^{2}$ Member, IEEE
}

[3] with the additional benefits of being particularly robust, thanks to a loop closure, and accurate across all the software platforms.

In this paper we propose to combine the usage of a CNN for detecting objects in the image frame as bounding boxes and the VIO to estimate the state of the mobile device by estimating the visual feature in the 3D space. This only requires a monocular camera and an Inertial Measurement Unit (IMU). We will call Bounding Box State Estimator (BBSE) the system we designed to overcome the Acc-Acc tradeoff. We can associate the 2D bounding boxes corners with the 2D features which 3D position is precomputed by the VIO system in order to track the bounding box in $3 \mathrm{D}$ and re-project its corner in the camera frame using the VIO pose when the system accelerates. We consider that at constant velocity the delay occurring by the neural network can be handle by classic computer vision tools so even if we provide a solution which works for constant speed, we particularly aim at compensating the detection CNN delay in the specific and more challenging case of acceleration and deceleration.

The resulting architecture automatically tracks objects and their bounding boxes through successive frames. We design this architecture to make it lightweight and ready-to-use. We chose VINS-Mono because it provides on-the-fly calibration of sensors, includes a pseudo sensor-synchronization and a failure detection system. YOLO is one the fastest object detector in the state-of-art and runs with minimal computation powered devices such as mobile phones. We emphasize that our architecture is not dependent on YOLO: any detector could be used instead. Particularly, those detectors that have inconsistent detection through time would benefit automatically from the tracking property of our system. Furthermore, this system is therefore not restricted to the detection of a specific kind of object as it is only dependent on the chosen detector. Lastly, although lightweight, the resulting product is adept at multitasking as it outputs robot pose estimation, object detection and tracking concurrently.

This paper is organized as follows. In Section 2, we review related works. In Section 3, we describe our architecture and the strategy to conjointly fuse object detection and the reprojection method. In Section 4, we explain the implementation condition and show our results.

\section{RELATED WORK}

\section{A. Object detection in $3 D$}

The real-time object detection algorithms have been used for many different practices. We will focus on mentioning their application for inferring the 3D information, for 
tracking and for high-rate real time detection. First, YOLO detection was mapped from a 2D detection to a 3D detection in [5] implemented for a camera system without any robotic mobile support.

Between tracking and mapping field of research, object pose estimation has also been researched with [14][19] or without neural networks [7]. However, this latter method were not developed in the perspective of a robotic object detection but rather for environment mapping like Semantic SLAM does. Semantic SLAM is heavy in computation, and for example [8] had its running time process exceeding 1 second. Semantic mapping also performs object detection as described in [15]. From a 2D detection performed by a CNN, they reconstruct a $3 \mathrm{D}$ bounding box. For the $3 \mathrm{D}$ reconstruction and further tracking of these inferred 3D bounding box, [15] uses multiple cameras working together. OpenPTrack [16] or SimTrack [14] also use multiple camera system or RGB-D in order to estimate the pose of the object. One of the greatest concerns in this field has been to improve metric scale accuracy [9]. The detected object needs to have been shaped beforehand with Computer Aided Design (CAD) in order to be added on the map or even to substitute the classic feature extraction approach for loop-closing improvement [10]. The first one to have used a monocular camera with CNN detection considered as features [11] has considered only fixed object on a mobile base like us, but they have focused their work on mapping a 3D world map for specific target and not for tracking or detection. Besides, their models contain class specific priors. For example, already shaped CAD model, unlike our model, which aims at a continuous detection with no prior knowledge about the CNN classes. They mention as well that deleting these constraints would forgo the ability to operate in real time. With our proposed method, no CAD or previous 3D shaping has been done, and we only rely on $2 \mathrm{D}$ detection without any mapping or accurate metric scale considerations.

\section{B. Tracking systems}

The architecture for "model-based tracking" enables the estimation of 3D object detection, but to our knowledge, no work has been done in this field to link two autonomous systems such as a CNN and VINS altogether. Most of these methods use visual features such as ORB[28] or region-based visual bundle adjustment where the first detection is supposed to be correct as in [19]. This paper is very close from our work in terms for 2D-3D matching. Nevertheless they do not rely aim at improving their detection support unlike us. In this paper we do not expect any good accuracy from our 3D estimation, but we expect an accurate reproduction to make our detection tool more robust.

\section{ARCHITECTURE}

\section{A. System Overview:}

Our system is an intermediary system between VINSMono [20] and YOLO [1] running independently in parallel.

First, the VIO system needs to be initialized with sufficient movement acquisition and processed features. Once the robot state estimator is running correctly, our BBSE system will be triggered by a $\mathrm{CNN}$-detected bounding boxes. One state of the BBSE corresponds to one real object to detect. If the $\mathrm{CNN}$-detection is occurring frequently enough on consecutive frames, the BBSE state will consider it as an object to track: it will then provide an accurate, robust and smooth detection along the acquired camera frames, despite acceleration of the robot. Otherwise, it will be discarded as a noisy unreliable $\mathrm{CNN}$-detection.

In order to perform accurate object detection and tracking, our BBSE creates three possible detection for the same object. If the robot is moving slowly or immobile, we will rely on the $\mathrm{CNN}$-detection. In between the $\mathrm{CNN}$-detected frames, we update our BBSE prediction by making use of optical flow [21] or a feature matching techniques to correct the delays in order to keep tracking the bounding box. Finally, if the robot starts to accelerate, we will re-project the $3 \mathrm{D}$ triangulated bounding box in the camera frame.

Therefore, any rigid objects with arbitrary shapes could be tracked as long as they are recognized by the CNN system and the VIO is not failing into estimating the state. Our system relies strongly on these two systems to work.

We will now define the notation used throughout this paper. We consider the bold notation as vectors: ${ }^{G} \mathbf{x}_{k}$ is a $3 \mathrm{D}$ vector in the $k$ frame described the $\mathrm{G}$ frame of reference. We consider ${ }^{G}(),{ }^{I}(),{ }^{F}(),{ }^{C}()$ respectively as the World, IMU, image Frame and Camera frames of references. ${ }^{G} \mathbf{X}$ is the vector containing the $3 \mathrm{D}$ estimation of the four corners of a bounding box in $G$ frame of reference. We use both Hamilton quaternion for the state and rotation matrix to represent rotation. In particular, $\mathbf{q}_{I}^{W}$ and $\mathbf{p}_{I}^{W}$ are the rotation and translation from body frame to world frame. We consider the IMU frame as the body frame. We will use the terms "CNN-detection" to mention CNN detected bounding box, "3D-detection" to describe the re-projection result of the $3 \mathrm{D}$ bounding box and "matched detection" for the result of the feature matching methods.

The structure of our system is shown in Fig. 1.

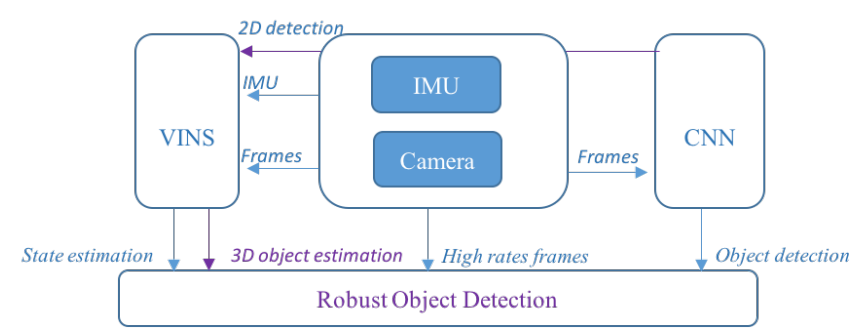

Fig. 1. We highlight in blue the fusion of the existing architectures, in purple we highlight our contribution.

\section{B. Calibration}

Calibration and sensor-synchronization are of crucial importance for any 6-dof state-estimator to run with robustness and accuracy [26]. Nevertheless to initialize these parameters requires advanced knowledge. In order to make our BBSE 
system simple to use, we take advantage of the online calibration provided by VINS-Mono. The extrinsic (Camera/IMu transformation), intrinsic (camera sensor parameters) and the time delay (Camera/IMU delay) can be given as approximations or not given at all to the system. These parameters will be refined and corrected automatically when starting the state estimator system [20]. Giving approximation beforehand makes its initialization faster when starting the detection process. It also prevents the system to fail running because of inaccurate parameters.

\section{Fusing $3 D$ and $2 D$ information}

Our approach relies on a continuous interaction between VINS real world information, the real time camera frame flow and the delayed CNN detection. We have designed a Bounding Box State Estimator for dealing with the information flow given by YOLO and VINS at different times in different frames of reference. The BBSE is composed of states. One state corresponds to one physically existing object in the real world. This BBSE tracker creates three possible detection: the CNN detection, 3D-detection or matched detection, as Fig. 1 illustrates. One state is made as shown is Equation (1).

$$
\begin{aligned}
s & =\left[\mathbf{x}_{\mathbf{k}}, \lambda,{ }^{G} \mathbf{X},{ }^{F} \mathbf{X}^{\text {cur }}\right] \\
\lambda & =\left[\lambda_{t l}, \lambda_{t r}, \lambda_{b l}, \lambda_{b r}\right] \\
{ }^{G} \mathbf{X} & =\left[{ }^{G} \mathbf{c}_{\mathbf{t l}},{ }^{G} \mathbf{c}_{\mathbf{t r}},{ }^{G} \mathbf{c}_{\mathbf{b l}},{ }^{G} \mathbf{c}_{\mathbf{b r}}\right]
\end{aligned}
$$

With $\lambda$ the vector of depth, $\mathbf{x}_{\mathbf{k}}$ is the robotic state inferred by VINS as detailed in Equation (4) at frame $\mathrm{k},{ }^{P} \mathbf{X}$ is the bounding corners vector described in $P$ frame of reference, considered in the cur current frame. The state also takes the parameters of the VIO system, that are necessary to perform the projections between the world, the IMU and the camera frame (such as intrinsic and extrinsic parameters).

\section{Building the 3D-bounding box in the world frame}

The VINS-Mono VIO system uses a sliding window and non linear graph optimization algorithm [20] to estimate a state vector defined as follows.

$$
\begin{aligned}
\mathbf{x} & =\left[\mathbf{x}_{\mathbf{0}}, \mathbf{x}_{\mathbf{1}}, \ldots \mathbf{x}_{\mathbf{n}}, \mathbf{x}_{\mathbf{c}}^{\mathbf{b}}, \lambda_{0}, \lambda_{1} \ldots \lambda_{m}\right] \\
\mathbf{x}_{\mathbf{k}} & =\left[{ }^{G} \mathbf{p}_{b_{k}},{ }^{G} \mathbf{v}_{b_{k}},{ }^{G} \mathbf{q}_{b_{k}}, \mathbf{b}_{\mathbf{a}}, \mathbf{b}_{\mathbf{g}}\right], k \in[0, n] \\
\mathbf{x}_{\mathbf{c}}^{\mathbf{b}} & =\left[\mathbf{p}_{c}^{I}, \mathbf{q}_{c}^{I}\right]
\end{aligned}
$$

Where $\mathbf{x}_{\mathbf{k}}$ is the IMU state when the $k^{\text {th }}$ image is captured. This state contains position, velocity and orientation of the IMU in the world frame as well as acceleration bias and gyroscope bias in the IMU body frame. $n$ is the total number of keyframes and $m$ is the total number of features we track in the sliding window. Finally, $\lambda_{i}$ is the inverse depth of the $i^{t h}$ feature from the first observation of this feature in the sliding window. The estimation of the IMU state of the robot which is performed, is beyond the scope of this paper.

We extract the information of the triangulation performed inside VINS to estimate the depth metric for the triangulation of our CNN-detected bounding box corners ${ }^{G} \mathbf{X}$. Indeed, the object detected by the neural network is likely to be contrasted with a particular shape or texture: its saliency makes it likely as well to be detected by the feature detector which performs the triangulation. The triangulation is processed in VINS for all the features in the current sliding windows with the Least-Squares Triangulation method. The estimated depth metric that we associate to each corner is updated as the same time as the the sliding window.

To find the inverse depth of the CNN-detected corners from the computed VINS feature, we look for the closest VINS 2D-feature from each corner, located inside the bounding box.

$$
\min _{j \in[1, m]}\left(\left\|{ }_{k}^{F} \mathbf{X}_{\mathbf{i}}-{ }^{F} \mathbf{p}_{\mathbf{k}}^{\mathbf{j}}\right\|\right), i \in[1,4], k \in[0, n]
$$

For every image frames $F$ where we track a bounding box, we seek the minimum value of Equation (7) for each of the four corners $i$ of one bounding box and all the detected and triangulated features in this frame. Once we have found which 2D-CNN corner can be associated with which 2DVINS feature, we retrieve the VINS feature inverse depth $\lambda_{i}$ and compute the corresponding 3D-bounding box in the World frame as detailed as follows.

$$
\begin{aligned}
{ }_{0}^{G} \overline{\mathbf{X}} & ={ }_{0}^{G} \mathbf{X} \lambda \\
{ }^{G} \mathbf{X} & ={ }_{0} R_{I}^{G}\left({ }_{0} R_{C}^{I}{ }_{0}^{G} \overline{\mathbf{X}}+{ }_{0} p_{C}^{I}\right)+{ }_{0} p_{I}^{G}
\end{aligned}
$$

After we associate each corner to a feature of the sliding window and its inverse depth, we can compute ${ }_{0}^{G} \overline{\mathbf{X}}$ containing the position of the corners $c_{i}$ of a bounding box $c$ in the World frame $\mathrm{G}$.

\section{E. Re-projection of the 3D-detection on the camera frame}

From the VIO estimator, we retrieve the current position and orientation in $\mathbf{x}_{\mathbf{k}}$ of the robot, as well as both extrinsic parameters $R_{I}^{C}$ (rotation) and $p_{C}^{I}$ (relative position) of the camera toward the IMU. If more than three corners of a bounding box could have been inferred in the world frame, we consider the re-projection as a valid means for inferring this bounding box location in the camera frame. This enable to project the 3D-bounding box with Equation (5).

$$
\begin{aligned}
{ }^{I} \mathbf{x} & =q_{G}^{I}\left({ }^{G} \mathbf{c}-p_{I}^{G}\right) \\
{ }^{C} \mathbf{x} & =R_{I}^{C}\left({ }^{I} \mathbf{x}-p_{C}^{I}\right) \\
{ }^{F} \mathbf{x} & =\left(\frac{{ }^{C} \mathbf{x} \cdot \mathbf{x}_{\mathbf{u}} f_{u}}{{ }^{C} \mathbf{x} \cdot \mathbf{z}_{\mathbf{u}}}+c_{u}, \frac{{ }^{C} \mathbf{x} \cdot \mathbf{y}_{\mathbf{u}} f_{v}}{{ }^{C} \mathbf{x} \cdot \mathbf{z}_{\mathbf{u}}}+v_{u}\right)
\end{aligned}
$$

In these equations, we transform successively a corner described in the world frame as a $3 \mathrm{D}$ object ${ }^{G} \mathbf{c}$, into ${ }^{F} \mathbf{x}$ in a image frame $F$ pixel, with $\mathbf{x}_{\mathbf{u}}, \mathbf{y}_{\mathbf{u}}, \mathbf{z}_{\mathbf{u}}$ unit vectors, and the monocular camera intrinsic parameters $c_{u}, v_{u} . f_{u}, f_{v}$.

\section{F. Speed-adapted inference strategy}

Our approach is fully relying on the reprojection system when the system is briskly accelerating or decelerating, because $\mathrm{CNN}$ detection are highly inaccurate in that case. Nevertheless for soft acceleration or constant speed, we use 
the $\mathrm{CNN}$ detection to match the already inferred and tracked bounding boxes with the new $\mathrm{CNN}$-detection we receive. The traditional approach uses the Hungarian algorithm with an Intersection Over Union (IOU) cost metric or a Kalman filter [18] as an association metric.

For our BBSE system, we first decided to adopt a speedadapted strategy. We first attribute to each of our BBSE state one of the three computed bounding boxes: the one inferred by the $\mathrm{CNN}$, by the feature matching or by the re-projection. Then, we use the VIO estimator to retrieve the velocity of the mobile system ${ }^{G} \mathbf{v}_{b_{k}}$, from this estimated speed. Finally by changing the IOU threshold between the new CNN detected bounding box and the three current states of the BBSE state, we can decide which method we rely the most on.

To perform it, we designed empirically three modes and the IOU threshold as we detail in Table 1. This table summarizes our strategy when the robot is moving. These modes are divided in slow (speed in all direction less than $0.4 \mathrm{~m} / \mathrm{s}$ ), medium (speed in at least one direction between 0.4 and $0.7 \mathrm{~m} / \mathrm{s}$ ) and fast (speed of at least one direction more than $0.7 \mathrm{~m} / \mathrm{s}$ ) movement. We set these values experimentally by finding the speeds above which the CNN or feature matching system is lightly or completely failing. In these latter case, we ask our reprojection system to take over and infer the tracked bounding box without relying anymore on the CNN. Forbidden mode means that we will not take this inference as the final inference, restricted mode means that the IOU threshold of the inferred bounding box and the newly detected is low and priority means the IOU threshold is very high so we are likely to track our previous estimation with the new inference value.

\section{TABLE I}

SPEED STRATEGY AND DESIGN OF PRIORITY FOR THE UPDATE OF TRACKING BOUNDING BOX STATE

\begin{tabular}{|l|c|c|c|}
\hline & Low Speed & Medium Speed & High Speed \\
\hline Feature Matching & Restricted & Priority & Restricted \\
\hline CNN & Priority & Restricted & Forbidden \\
\hline Re-projection & Forbidden & Restricted & Priority \\
\hline
\end{tabular}

\section{G. Acceleration-adapted inference strategy}

TABLE II

ACCELERATION STRATEGY

\begin{tabular}{|c|c|c|}
\hline & Low to Medium & Medium to High \\
\hline Acceleration & CNN+Reproj & Reproj \\
\hline \hline & High to Medium & Medium to Low \\
\hline Deceleration & Reproj & CNN+Reproj \\
\hline
\end{tabular}

Table 1 illustrates the speed strategy to counterbalance the delay in constant velocity case while Table 2 summarizes our strategy towards the Acc-Acc tradeoff. We combine these two strategies to have a system robust to any kind of movement. If the robot accelerates from medium to high speed and decelerates from high speed to medium we only rely on the re-projection without taking in account the CNN values as stated before. For medium acceleration, we take the IOU between our reprojection system and the CNN with low threshold. Besides, if the robot has a high speed, CNN detection will not be taken into account and we will rely essentially on the re-projection or the optical features. The IOU threshold with the CNN is set as small as the device is fast, since the detection are less likely to overlap.

The acceleration strategy has priority over the speed strategy if we consider the robot accelerating/decelerating. If the device is too fast, we impeded the creation of new states in order to avoid noisy detection created by the CNN.

In case of slow speed motion, we apply as a guarantee the Hungarian Algorithm on the IOU for a robust matching. If the bounding box is still unmatched after the IOU and Hungarian Matching steps, we create a new state symbolizing a new object detected in the World map. If the number of successive detection has not been detected, the state is discarded.

\section{IMPLEMENTATION}

\section{A. Environment}

Our system was designed for indoor robotics. Because of the lack of an appropriate and complete dataset for our application, we decided to perform the experimentation ourselves. We first decided to run a simulated environment to evaluate our algorithm. We use Gazebo [23] where we simulated the AscTec Hummingbird drone [22] equipped with the VI-Sensor [27] and controlled with RPG Quadrotor Control [29]. We display our system state and result in Rviz [24] The simulation and the VINS-Mono transformed in the VINS-YOLO tracker was run on a desktop computer 7.7 GiB, Intel Core i3-6100 CPU @ 3.70GHz x 4, equipped with a GeForce GTX $1050 \mathrm{Ti}$ on Ubuntu 16.04. All visual captors and the neural network are meant to run on the mobile plateform, we chose the Jetson TX2 Nvidia Tegrax2, which has an ARMv8 Processor rev3 x 4 ARMv8. Since we aim at robotic application, the Jetson is symbolizing the future real robot which is able to send the results to other fixed platforms like our desktop computer.

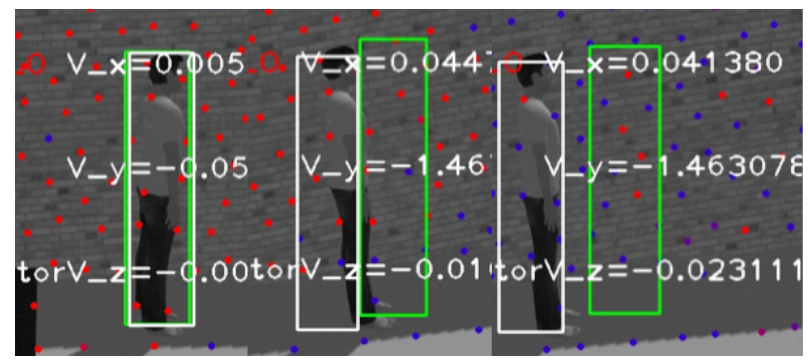

Fig. 2. Three consecutive frames showing the acceleration of a drone YOLO bounding box in green, suffers from a delay, while the white one, from our tracker, remains accurate. Red points are the features of the current frame which are triangulated. In white, the inferred speed of the system.

In this paper we will use ROS [25] to run the simulation and to carry the information topic to each thread accordingly to the architecture illustrated in Fig. 1. 


\section{B. Delay considerations}

Due to the simulation computation requirement, all the topics are received with a frequency of $3-4 \mathrm{~Hz}$, which corresponds to the YOLOv3 processing time. In this configuration, we have measured the delay between two callbacks for the three inputs: raw frames $50 \mathrm{~ms}$, VINS features computation $60 \mathrm{~ms}$ and YOLO detection 200ms. Since the YOLOv3 average processing time on ROS is $200-250 \mathrm{~ms}$, it has approximately 4-5 frames of delays since ROS acquire a new frame every 50ms approximately. The feature computation has approximately the same processing rate as the frames, therefore we do not take in account this delay. We notice that, since the UAV can often reach speeds such as $0.5 \mathrm{~m} / \mathrm{s}$ when initiating a movement, the existence of $200 \mathrm{~ms}$ of delay from the CNN implies that the drone use the detection of the frame of its state from $10 \mathrm{~cm}$ before. Our BBSE system will help correcting this.

\section{Experience conditions}

We tested our system on 3 sequences made in the simulated world, where we moved our drone around a human. Video $\mathrm{A}$ is $2 \min 40$ s with a man standing against a wall and we only used the speed-strategy, Video B is 1 min $46 \mathrm{~s}$ with same configuration to which we added the acceleration/deceleration prediction system. Finally, Video C lasts 2 min 18 s with a human in front of a window and further from the robot than in Video A and B. We set the ground-truth bounding box manually for every frames, which has limited the number of our testing data video. Our sliding windows was set to 24 frames, the variable IOU threshold is set at lowest 0.1 (priority mode) otherwise 0.4 (restricted mode) for matching current and new detection. We chose ORB [28] as feature matcher in case the Lucas-Kanade optical flow [21] failed (less than $50 \%$ of the current and predicted bounding box does not overlap). For evaluating the success rate of our system we created an accuracy average function which take for every frames the IOU rate of the ground truth object detection with one of the system: YOLO only detection or our BBSE tracker:

$$
\begin{gathered}
\sum_{k=1}^{N} \frac{\mathbb{1}_{I O U}\left(\mathbf{x}_{k}^{s y s}, \mathbf{x}_{k}^{g t}\right)}{N} \\
\mathbb{1}_{\text {IOU }}\left(\mathbf{x}_{k}^{s y s}, \mathbf{x}_{k}^{g t}\right)=\left\{\begin{array}{l}
1 \text { if } \operatorname{IOU}\left(\mathbf{x}^{s y s}, \mathbf{x}^{g t}\right) \geq t \\
0 \text { otherwise }
\end{array}\right.
\end{gathered}
$$

With $N$ the number of frames considered in one video, $\mathbf{x}_{k}$ the bounding box vector in the $k$ frame, $t$ the IOU threshold, $\mathbb{1}_{I O U}$ the function to create the success rate as an average accuracy value comparing YOLO detection and BBSE with ground truth, sys is in $\{Y O L O, B B S E\}$. For each frame of the test videos, we manually determined $\mathbb{1}_{I O U}$ for separately YOLO and BBSE systems according to the two thresholds. We consider the detection to have failed (1 if $\operatorname{IOU}\left(\mathbf{x}^{s y s}, \mathbf{x}^{g t}\right)=0$ ) if the IOU of the inferred bounding box is under the threshold set.

\section{Results}

The result for the video A, B and C are displayed in Table 3. We take two IOU thresholds between the system detection and the ground truth: $t=0.7$ for precise detection and $t=0.4$ for slightly off detection (values in parenthesis in the Table 3 ). For each system, we consider either the average on all the frames of the video (All), either the frames where the robot moves (M) or either considering only the frames when the robot accelerating or decelerating (Acc) which includes three frames before and after starting or stopping any movement.

TABLE III

SucCess RATE of THE DETECTION ON VIDEO SEQUENCES A,B AND C

\begin{tabular}{|c|c||c|}
\hline & YOLO & BBSE \\
\hline & All & All \\
\hline A & $0.83(0.91)$ & $\mathbf{0 . 8 7}(0.91)$ \\
\hline B & $0.76(0.82)$ & $\mathbf{0 . 8 7}(0.93)$ \\
\hline C & $0.79(0.87)$ & $\mathbf{0 . 9 4}(0.97)$ \\
\hline
\end{tabular}

\begin{tabular}{|c|c|c||c|c|}
\hline & YOLO & YOLO & BBSE & BBSE \\
\hline & M & Acc & M & Acc \\
\hline A & $0.33(0.67)$ & $0.40(0.60)$ & $0.5(0.7)$ & $0.69(0.76)$ \\
\hline B & $0.41(0.57)$ & $0.42(0.66)$ & $0.65(0.84)$ & $0.66(0.88)$ \\
\hline C & $0.32(0.57)$ & $0.53(0.71)$ & $0.88(0.93)$ & $0.86(0.93)$ \\
\hline
\end{tabular}

As Table 3 shows in bold, the BBSE method has a better accuracy for both IOU thresholds for all videos. Videos $\mathrm{A}$ and $\mathrm{B}$ have the same scene configuration but different velocities and trajectories which explains the differences in the YOLO detection values. We justify that we got the same BBSE success rate for both video because the moment when the robot is immobile or moving are different for both videos. Nevertheless, we can see that Video B for which we added our Acceleration strategy is performing better for the Moving (M) or Acceleration (Acc) phases than in Video A where this system was off. Indeed, BBSE succeeds on improving the results for moving phases. It has more than $10 \%$ more accuracy than YOLO for the whole Video $\mathrm{C}$ and more than $50 \%$ more of accuracy if we consider the detection during moving phases. The success rate of the detection during the acceleration (Acc) is always more than $20 \%$ better with BBSE than pure YOLO. The reason why the success rate of YOLO is greater is acceleration and deceleration phase is because we take into account before the movement starts until few frames it started. This includes frames where the CNN detection is still accurate since the robot is not yet moving.

\section{E. Discussion and future improvements}

Firstly, since the re-projection system aims at improving the detection system and not the inertial system, it states VINS like ground truth. This means that a failure of VINS implies the failure of our re-projection. VINS is prone to fail as it accumulates error and when the state estimator diverges, the failure system is triggered and the BBSE system as well is reinitialized. The protection we implemented against VINS failure was to detect at least three corners to create the 3D-detection, which is not robust enough if VINS fails. 
Developing a system which detect the VINS failures and relies only on optical flow and CNN until VINS is fixed would be one of the next steps of our work.

Secondly, we based our strategy on a robust CNN detection. Nevertheless if the CNN fails at detecting an object on consecutive frames the BBSE tracker for this object is discarded. More than improving the accuracy of the detection along movement, we could add a robust tracking which do continue tracking object we do not receive any $\mathrm{CNN}$ detection for more frames.

Thirdly, in order to re-project our 3D-bounding box in the image frame, we only used the information on the top left corner and bottom right corner of the retrieved bounding box. An implementation of an optimization algorithm to compute more accurate 3D-detection with the information of the fourth detected corners already retrieved by our tracker could improve the robustness as well.

\section{CONCLUSION}

We have presented a novel method to improve the detection of fixed object in the context of a moving device regarding to the accuracy-acceleration tradeoff. Our method enables simultaneous real-time self-localization, object detection and tracking. We built our system with VINS-Mono and YOLO both open source algorithms and ready-to-use. Furthermore any detector can replace YOLO. For improvement, the next step of our system would be to implement it on a real robot with CNN running customized dataset.

\section{REFERENCES}

[1] Redmon, Joseph and Ali Farhadi. "YOLOv3: An Incremental Improvement." 2018

[2] Apte, M. "YOLO Net on iOS." (2017).

[3] Delmerico, Jeffrey \& Scaramuzza, Davide. "A Benchmark Comparison of Monocular Visual-Inertial Odometry Algorithms for Flying Robots." (2018).

[4] Pedoeem, Jonathan \& Huang, Rachel. "YOLO-LITE: A Real-Time Object Detection Algorithm Optimized for Non-GPU Computers." (2018).

[5] Simon, Martin et al. "Complex-YOLO: Real-time 3D Object Detection on Point Clouds.". 2018.

[6] Alex Krizhevsky, Ilya Sutskever, and Geoffrey E. Hinton. 2012. "ImageNet classification with deep convolutional neural networks." In Proceedings of the 25th International Conference on Neural Information Processing Systems - Volume 1 (NIPS'12), F. Pereira, C. J. C. Burges, L. Bottou, and K. Q. Weinberger (Eds.), Vol. 1. Curran Associates Inc., USA, 1097-1105.

[7] Henning Tjaden, Ulrich Schwanecke and Elmar SchÃúmer, "RealTime Monocular Pose Estimation of 3D Objects Using Temporally Consistent Local Color Histograms". 2017 IEEE International Conference on Computer Vision (ICCV), 2017, p.124-132.

[8] Sudeep Pillai and John Leonard, "Monocular SLAM Supported Object Recognition", Robotics: Science and Systems 2015

[9] Edgar Sucar and Jean-Bernard Hayet, "Probabilistic Global Scale Estimation for MonoSLAM Based on Generic Object Detection", Int. Workshop on Visual Odometry, CVPR 2017.

[10] S. L. Bowman, N. Atanasov, K. Daniilidis and G. J. Pappas, "Probabilistic data association for semantic SLAM," 2017 IEEE International Conference on Robotics and Automation (ICRA), Singapore, 2017, pp. 1722-1729.

[11] J. Dong, X. Fei and S. Soatto, "Visual-Inertial-Semantic Scene Representation for 3D Object Detection," 2017 IEEE Conference on Computer Vision and Pattern Recognition (CVPR), Honolulu, HI, 2017, pp. 3567-3577
[12] Zhen Zeng, Yunwen Zhou, Odest Chadwicke Jenkins and Karthik Desingh, "Semantic Mapping with Simultaneous Object Detection and Localization", 2018 IEEE/RSJ International Conference on Intelligent Robots and Systems (IROS), 2018, p.911-918.

[13] J. Redmon, S. Divvala, R. Girshick and A. Farhadi, "You Only Look Once: Unified, Real-Time Object Detection," 2016 IEEE Conference on Computer Vision and Pattern Recognition (CVPR), Las Vegas, NV, 2016, pp. 779-788.

[14] K. Pauwels and D. Kragic, "SimTrack: A simulation-based framework for scalable real-time object pose detection and tracking," 2015 IEEE/RSJ International Conference on Intelligent Robots and Systems (IROS), Hamburg, 2015, pp. 1300-1307.

[15] Li P., Qin T., Shen S. "Stereo Vision-Based Semantic 3D Object and Ego-Motion Tracking for Autonomous Driving". In: Ferrari V., Hebert M., Sminchisescu C., Weiss Y. (eds) Computer Vision - ECCV 2018.

[16] M. Munaro, F. Basso and E. Menegatti. "OpenPTrack: Open Source Multi-Camera Calibration and People Tracking for RGB-D Camera Networks." Journal on Robotics and Autonomous Systems, vol. 75, part B, pp. 525-538, Elsevier, 2016.

[17] Saeed Ranjbar Alvar and Ivan V. Bajic, MV-YOLO: Motion VectorAided Tracking by Semantic Object Detection, IEEE 20th International Workshop on Multimedia Signal Processing (MMSP), 2018

[18] Alex Bewley, ZongYuan Ge, Lionel Ott, Fabio Ramos and Ben Upcroft, "Simple Online and Realtime Tracking", 2016 IEEE International Conference on Image Processing.

[19] Qiu, Kejie et al. "Estimating Metric Poses of Dynamic Objects Using Monocular Visual-Inertial Fusion." 2018 IEEE/RSJ International Conference on Intelligent Robots and Systems (IROS). ,

[20] T. Qin, P. Li and S. Shen, "VINS-Mono: A Robust and Versatile Monocular Visual-Inertial State Estimator," in IEEE Transactions on Robotics, vol. 34, no. 4, pp. 1004-1020, Aug. 2018.

[21] B. D. Lucas and T. Kanade, "An iterative image registration technique with an application to stereo vision," in Proc. of the Intl. Joint Conf. on Artificial Intelligence, Vancouver, Canada, Aug. 1981, pp. 24-28.

[22] Furrer, Fadri \& Burri, Michael \& Achtelik, Markus \& Siegwart, Roland. (2016). "RotorS - A Modular Gazebo MAV Simulator Framework."

[23] N. Koenig and A. Howard, "Design and use paradigms for Gazebo, an open-source multi-robot simulator," 2004 IEEE/RSJ International Conference on Intelligent Robots and Systems (IROS) (IEEE Cat. No.04CH37566), Sendai, 2004, pp. 2149-2154 vol.3.

[24] Kam, Hyeong Ryeol \& Lee, Sung-Ho \& Park, Taejung \& Kim, ChangHun. (2015). "RViz: a toolkit for real domain data visualization". Telecommunication Systems.

[25] Quigley, Morgan \& Conley, Ken \& P Gerkey, Brian \& Faust, Josh \& Foote, Tully \& Leibs, Jeremy \& Wheeler, Rob \& Y Ng, Andrew. (2009). ROS: an open-source Robot Operating System. ICRA Workshop on Open Source Software.

[26] Ling, Yonggen et al. "Modeling Varying Camera-IMU Time Offset in Optimization-Based Visual-Inertial Odometry." ECCV (2018).

[27] Nikolic, J., Rehder, J., Burri, M., Gohl, P., Leutenegger, S., Furgale, P. T., \& Siegwart, R. (2014). "A Synchronized Visual-Inertial Sensor System with FPGA Pre-Processing for Accurate Real-Time SLAM." IEEE International Conference on Robotics and Automation (ICRA), Hongkong, China.

[28] Rublee, Ethan \& Rabaud, Vincent \& Konolige, Kurt \& Bradski, Gary. (2011). "ORB: an efficient alternative to SIFT or SURF." Proceedings of the IEEE International Conference on Computer Vision.

[29] M. Faessler, D. Falanga and D. Scaramuzza, "Thrust Mixing, Saturation, and Body-Rate Control for Accurate Aggressive Quadrotor Flight," in IEEE Robotics and Automation Letters, vol. 2, no. 2, pp. 476-482, April 2017. 